\title{
Design of T-Junction to Improve Splitting Process of Reagent in Gas-Liquid Microfluidics
}

\author{
Pin-Chuan Chen*, Ming-Han Wu and Yan-Ning Wang \\ Department of Mechanical Engineering, National Taiwan University of Science and Technology, \\ Taipei, Taiwan
}

(Received June 10, 2013; accepted October 1, 2013)

Key words: uniform reagent distribution, microfluidics, T-junction design, micromilling

Uniform reagent distribution is an important factor in a high-throughput microfluidic system, since it can significantly improve the user interface and simplify the reagent loading process. The uniformity of the reagent distribution depends on the geometrical design, roughness of the microchannel surface, and the surface property of the microchannel. The design of the T-junction significantly affects the uniformity of the reagent distribution, and this study focuses on the geometry design of this T-junction. Two microfluidic devices were manufactured with a micromilling machine: one was a standard T-junction and one was a modified T-junction with circular corners and a separator. Fifty experiments were performed in each microfluidic device. The results showed that the modified T-junction achieved better uniformity and reduced reagent loss during the reagent splitting process. The modified T-junction design can be applied in a high-throughput microfluidic device for rapid and uniform reagent distribution.

\section{Introduction}

Microfluidic technology has been in development for three decades since the first micro-gas chromatograph was reported in $1979,{ }^{(1)}$ which elucidated that a bio/chemical reaction can be realized on a small footprint with the benefits of lower reagent demand, faster reaction rate, minimized labor to reduce contamination, and the potential to integrate with other functional components as a micro-total-analysis system ( $\mu$ TAS). To further expand the power of a $\mu \mathrm{TAS}$, the idea of a high-throughput microfluidic system is developed and realized both in the research laboratory ${ }^{(2-5)}$ and for the commercial market. ${ }^{(6)}$

A medical technician usually follows the chemical/biochemical protocol to mix well multiple reagents inside a centrifuge tube with a pipet or a centrifuge machine, and then manually loads the reagent into multiple centrifuge tubes. To efficiently deliver the sample or reagent to the designed location in a high-throughput system, sample distribution chips $^{(7)}$ and a commercial reagent pipetting system were developed

*Corresponding author: e-mail: pcchen@mail.ntust.edu.tw 
or used. A commercial reagent pipetting system is an efficient tool for loading tiny amounts of multiple reagents into a microfluidic chip, but this system is not suitable for a miniaturized system because of the bulky mechanism required to achieve precise loading. To enhance the reagent loading system in a miniaturized high-throughput system, a reagent distribution microfluidic chip is developed. ${ }^{(7)}$ The key design inside a reagent distribution microfluidic device is the T-junction where the reagent is split into two daughter droplets. The aim of this study is to improve the performance of the reagent distribution device by optimizing the geometry of the T-junction. Through accurate control of the pneumatic pressure and careful design of the T-junction microchannel, the small volume of reagent inside the loading channel will be uniformly split into multiple droplets in the microfluidic device. A nanoliter microfluidic dispenser was fabricated to perform rapid and uniform reagent loading onto a plate. ${ }^{(8)}$ It provides an inexpensive and compact solution for liquid sample distribution with the accuracy within the range of nanoliters. This polydimethylsiloxane (PDMS) microfluidic device can load 32 independent drops of reagents into 32 microchambers simultaneously, each droplet being $115 \mathrm{nl}$ with a coefficient of variance $(\mathrm{CV})$ of less than $6 \%$.

\section{Materials and Methods}

The layout of a circular high-throughput microfluidic disk is shown in Fig. 1. There is a general reagent loading channel in the center and multiple distribution channels arranged radially from the center. The well-mixed chemical reagent is loaded into the reagent loading channel with a pipet owing to capillary force. A uniform pneumatic pressure is applied from the top of the reagent loading channel to push the uniformly distributed reagent into multiple distribution channels. Two types of T-junctions were designed for testing: a standard type [Fig. 2(a)] and a modified type [Fig. 2(b)]. To simplify the study process and focus attention on the design of the T-junction, both devices have a loading channel and only two distribution channels. The modified type has a circular corner and a separator to improve the reagent splitting process.

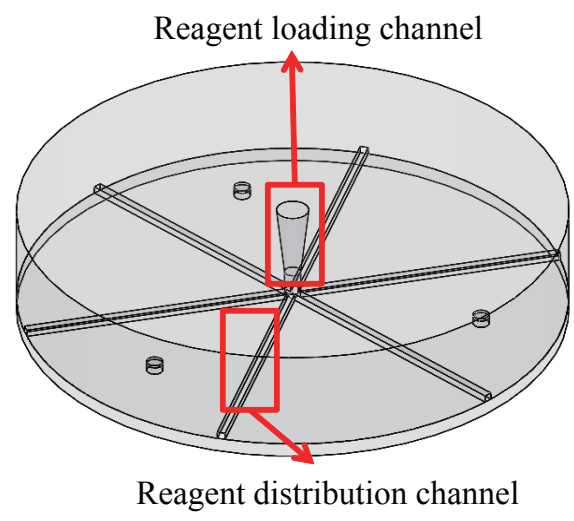

Fig. 1. (Color online) Microfluidic device designed to distribute the reagent from a single reservoir to six microchannels. 


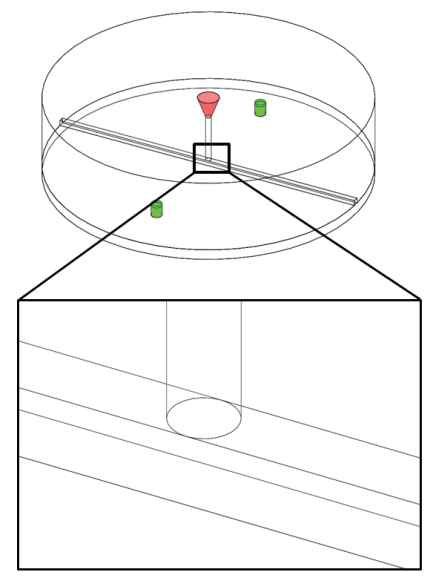

(a)

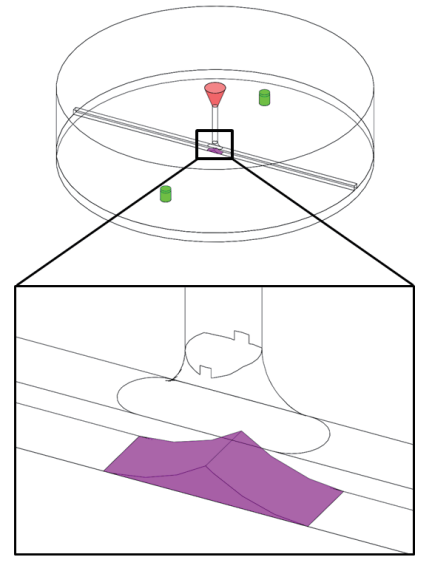

(b)

Fig. 2. (Color online) Two types of T-junctions were fabricated with a micromilling machine for reagent distribution experiments: (a) standard T-junction and (b) modified T-junction.

\subsection{Microfabrication of T-junction}

Two types of polymer microfluidic devices were manufactured with the micromilling machine. Four steps are required to manufacture a polymer microfluidic device for testing: (1) design drawing in a CAD file, (2) CAD file conversion into G-code for the micromilling controller, (3) micromilling, and (4) bonding. Less than $4 \mathrm{~h}$ is required to realize a ready-to-use chip for testing. A micromilling machine with five major components - a micromilling spindle (E3000c, Nakanishi, Japan), a laser noncontact tool setting system (NC4, Renishaw, United Kingdom), a numerical controller (M515i, LNC Technology Co. Ltd., Taiwan), a compressed air/oil coolant system, and a milling bit holder for tool exchange-was used in this study (Fig. 3). The micromilling bit used in chip fabrication was a 2-flute endmill with a diameter of $200 \mu \mathrm{m}$ (Taiwan Microdrill Co. Ltd., Taiwan).

\subsection{Measurement method}

The reagent was loaded into the loading channel [Fig. 4(a)] and a pneumatic force was applied from the inlet to uniformly distribute the sample into two distribution channels. A transparent projection film was printed out with a scale and attached to the back of the device. The scale was used to measure the uniformity of the split droplet by measuring the length of the droplet with a tool microscope [Fig. 4(b)].

\section{Results and Discussion}

Fifty experiments were realized in the two microfluidic devices, and the results are plotted in Fig. 5. Figure 5(a) shows the measured volumes of the daughter droplets in a standard T-junction device while Fig. 5(b) shows the measured volumes of the daughter droplets in the modified T-junction device. In the standard type, the average volume of 


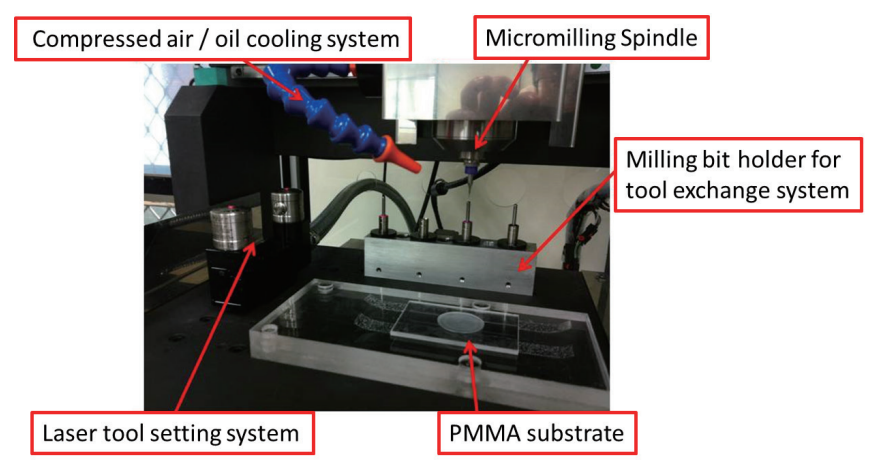

Fig. 3. (Color online) Micromilling machine used to fabricate polymer microfluidic devices.

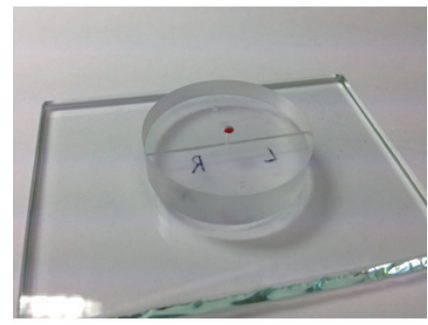

(a)

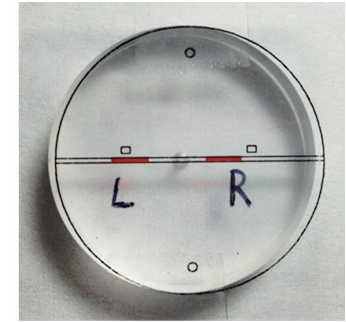

(b)

Fig. 4. (Color online) (a) A reagent was loaded in a loading channel for reagent splitting experiments. (b) The reagent was split into two daughter droplets after being pushed by a pneumatic system.

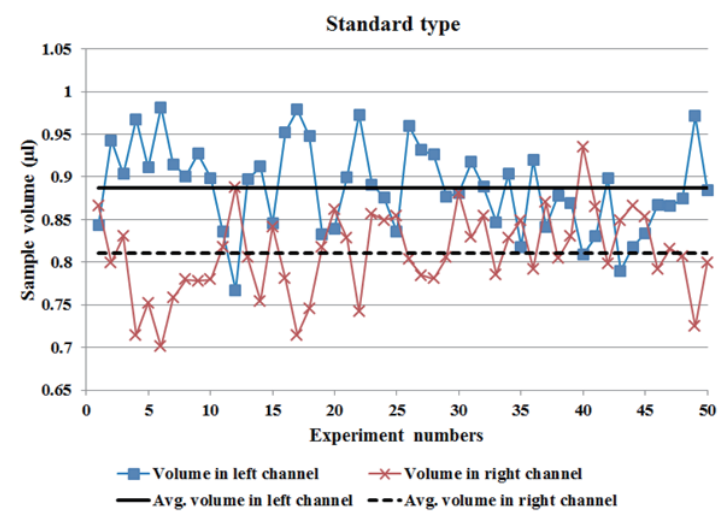

(a)

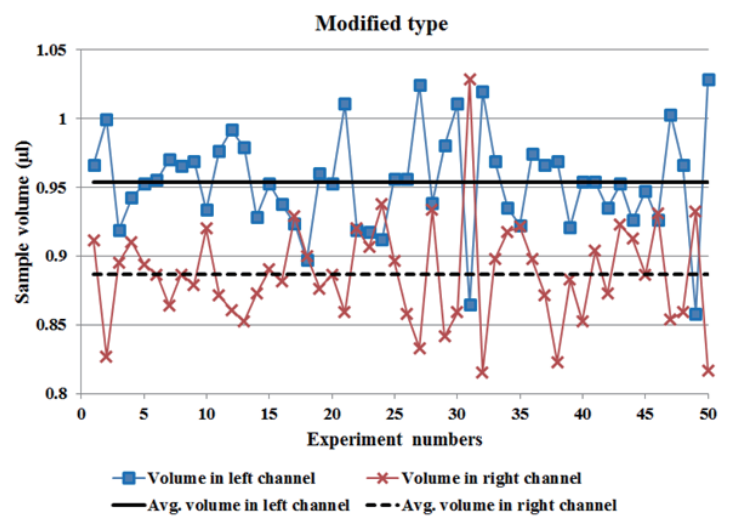

(b)

Fig. 5. (Color online) (a) Experiment results for standard T-junction. (b) Experiment results for modified T-junction. 
the left channel was $0.887 \mu \mathrm{l}$ and the average volume of the right channel was $0.810 \mu \mathrm{l}$. In the modified type, the average volume of the left channel was $0.953 \mu \mathrm{l}$ and the average volume of the right channel was $0.886 \mu \mathrm{l}$. The average volume difference between the two distribution channels in the standard device is $5.5 \%$ with an STD of $4.14 \%$, while the average volume difference between the two distribution channels in the modified device is $3.85 \%$ with an STD of $2.98 \%$. The average reagent loss is $0.306 \mu \mathrm{l}$ in the standard device and $0.164 \mu \mathrm{l}$ in the modified device during the splitting process. This loss might be caused by the roughness of the microchannel walls and the geometry of the microchannel.

\section{Conclusions}

Rapid and uniform reagent distribution is a critical factor in a high-throughput microfluidic disk. The geometrical design and substrate material properties played important roles in determining the performance of the reagent distribution device. PMMA is a mainstream material in microfluidics and it was used in our study. Micromilling was used to fabricate two types of polymer microfluidic devices for reagent distribution experiments: a standard T-junction and a modified T-junction with circular corner and separator. Fifty experiments were realized in the two devices, and the experimental results showed that the modified device achieved better uniformity, higher reliability, and less reagent loss during the splitting process.

\section{Acknowledgements}

This work was supported and funded by the National Science Council of Taiwan (NSC-102-2221-E-011-015) and Mechanical Engineering Department of National Taiwan University of Science and Technology (NTUST).

\section{References}

1 S. C. Terry, J. H. Jerman and J. B. Angell: IEEE Trans. Electron Devices 26 (1979) 1880.

2 B. M. Paegel, C. A. Emrich, G. J. Wedemayer, J. R. Scherer and R. A. Mathies: PNAS 99 (2002) 574.

3 C. A. Emrich, H. Tina, L. L. Medintz and R. A .Mathies: Anal. Chem. 74 (2002) 5076.

4 D. S-W. Park, P. C. Chen, B. H. You, N. Kim, T. Park, T. Y. Lee, P. Datta, Y. Desta, S. A. Soper, D. E. Nikitopoulos and M. C. Murphy: J. Micromech. Microeng. 20 (2010) 055003.

5 P. C. Chen, D. S. Park, B. H. You, N. Kim, T. Park, S. A. Soper, D. E. Nikitopoulos and M. C. Murphy: Sens. Actuators, B 149 (2010) 291.

6 T. O. Morrison, J. Hurley, J. Garcia, K. Yoder, A. Katz, D. Roberts, J. Cho, T. Kanigan, S. E. llyin, D. Horowitz, J. M. Dixon and C. J. H. Brenan: Nucl. Acid. Res. 34 (2006) e123.

7 D. S. Park, H. Wang, P. C. Chen, T. Park, N. Kim, B. H. You, D. E. Nikitopoulos, S. A. Soper and M. C. Murphy: 14th International Conference on Miniaturized Systems for Chemistry and Life Sciences 3-7 October 2010, Groningen, The Netherlands, 1226-1228.

8 J. Wang, Y. Zhou, H. Qiu, H. Huang, C. Sun, J. Xi and Y. Huang: Lab Chip 9 (2009) 1831. 